\title{
RIGIDITY FOR METRICS WITH THE SAME LENGTHS OF GEODESICS
}

\author{
Plamen Stefanov and Gunther Uhlmann
}

\begin{abstract}
We prove that we can recover a Riemannian metric in a bounded smooth domain in $\mathbb{R}^{3}$ up to an isometry which is the identity on the boundary, by knowing the lengths of the geodesics joining points on the boundary. We assume that the metrics are close to the euclidian metric $e$.
\end{abstract}

\section{Introduction and statement of the results}

Let $\Omega \subset \mathbb{R}^{3}$ be a bounded domain with smooth boundary $\Gamma=\partial \Omega$. Let $g(x)=\left(g_{i j}(x)\right)$ be a Riemannian metric in $\Omega$. Assume that $\bar{\Omega}$ is strictly convex with respect to $g$, i.e., for any two distinct points $x \in \bar{\Omega}, y \in \bar{\Omega}$ there is a unique geodesic joining $x$ and $y$ lying entirely in $\Omega$ with possible exception the endpoints $x$ and $y$. Let $d_{g}(x, y)$ denote the geodesic distance between $x$ and $y$. The inverse problem we address in this paper is whether we can determine the Riemannian metric $g$ knowing $d_{g}(x, y)$ for any $x \in \Gamma, y \in \Gamma$. It is easy to see that $g$ cannot be determined from this information. We have $d_{\psi^{*} g}=d_{g}$ for any diffeomorphism $\psi: \bar{\Omega} \rightarrow \bar{\Omega}$ that leaves the boundary pointwise fixed, i.e., $\left.\psi\right|_{\Gamma}=I d$, where $I d$ denotes the identity map and $\psi^{*} g$ is the pull-back of the metric $g$. R. Michel conjectured in [M1] that this is the only obstruction to uniqueness, namely if we have two Riemannian metrics $g_{1}, g_{2}$ with $\bar{\Omega}$ strictly convex with respect to both, and if

$$
d_{g_{1}}(x, y)=d_{g_{2}}(x, y) \quad \forall(x, y) \in \Gamma^{2},
$$

then there exists a diffeomorphism $\psi: \bar{\Omega} \rightarrow \bar{\Omega},\left.\psi\right|_{\Gamma}=I d$, so that

$$
g_{2}=\psi^{*} g_{1}
$$

The function $d_{g}$ measures the sojourn (travel) times of geodesics joining points of the boundary. In the case that both $g_{1}$ and $g_{2}$ are conformal to the euclidian metric $e$ (i.e., $\left(g_{k}\right)_{i j}=\alpha_{k} \delta_{i j}, k=1,2$ with $\delta_{i j}$ the Krönecker symbol), then the problem we are considering here is known in seismology as the inverse kinematic problem. In this case, it has been proven (see $[\mathrm{B}],[\mathrm{C}],[\mathrm{Mu}],[\mathrm{Mu}-\mathrm{R}]$ ) under

Received August 5, 1997.

The first author is partially supported by the Bulgarian Research Foundation, Grant MM 407. This work was done while P.S. was visiting the University of Washington.

The second author is partially supported by NSF Grant DMS-9705792 and ONR Grant N00014-93-1-0295. 
further restrictions on the metrics that if $d_{g_{1}}=d_{g_{2}}$, then $g_{1}=g_{2}$. In this case the diffeomorphism $\psi$ as in (1.2) must be the identity.

The conjecture (1.2) has been considered in [C], [Gr], [M1], [O] for general Riemannian manifolds with boundary under some assumptions on the curvature. In $[\mathrm{G}-\mathrm{N}]$ it discusses the two dimensional case in euclidian space. The linearized problem has been extensively studied in [Sh]. In this paper we prove the conjecture (1.2) under the condition that the metrics are close in an appropriate sense to the euclidian metric. More precisely, denote by $C_{(0)}^{k}(\Omega)$ the set of all $f \in C^{k}(\bar{\Omega})$ such that $\partial^{\alpha} f=0$ on $\partial \Omega$ for $|\alpha| \leq k$. Then we have

Theorem 1.1. Suppose that $g_{1}$ and $g_{2}$ are two metrics satisfying (1.1). Then there exists $\varepsilon>0$, such that if

$$
g_{m}-e \in C_{(0)}^{12}(\Omega), \quad\left\|g_{m}-e\right\|_{C^{12}(\bar{\Omega})}<\varepsilon, \quad m=1,2,
$$

then there exists a $C^{11}$ diffeomorphism $\psi: \bar{\Omega} \rightarrow \bar{\Omega}$ such that $\left.\psi\right|_{\Gamma}=I d$ and $\psi^{*} g_{1}=g_{2}$.

We also remark that there are two closely related inverse problems. Suppose we have a Riemannian metric which is the euclidian metric outside a compact set. The inverse scattering problem for metrics is to determine the Riemannian metric by measuring the scattering operator (see $[\mathrm{G}]$ ). A similar obstruction to (1.2) occurs in this case with $\psi$ equal to the identity outside a compact set. It was proven in $[\mathrm{G}]$ that knowing the scattering operator one can determine, under some non-trapping assumptions on the metric, $d_{g}$ on the boundary of a large ball.

One can consider also the hyperbolic Dirichlet-to-Neumann map $\Lambda_{g}$ associated to the wave equation $\left(\partial_{t}^{2}-\Delta_{g}\right) u=0$ with $g$ a Riemannian metric on $\bar{\Omega}$, with $\Omega$ being a bounded domain with smooth boundary and $\Delta_{g}$ being the LaplaceBeltrami operator (see $[\mathrm{Sy}-\mathrm{U}]$ ). It was proven in $[\mathrm{Sy}-\mathrm{U}]$ under the assumption of no caustics in $\bar{\Omega}$ for $g$, that knowing $\Lambda_{g}$, one can recover $d_{g}$.

Our proof of Theorem 1.1 relies on deriving an identity (see (2.10)) for the difference of the metrics and working in suitable chosen coordinates. The linearized version of the identity at the euclidian metric gives, roughly speaking, that the integrals along the geodesics (lines in the linear case) of the difference of the two metrics is zero (see (2.12) and (2.14)). Then one concludes that the metrics are the same in those coordinates by inverting the X-ray transform. This is done in section 2 .

In section 3 we carry out the proof of Theorem 1.1 by using a perturbation argument that leads to the inversion of a Fourier integral operator.

We remark that we assume in Theorem 1.1 that the metrics coincide to order 12 with the euclidian metric at the boundary. We only need to assume that in some coordinates. In [M1] Michel proved that the assumption (1.1) implies that the derivatives of the metrics up to order 2 coincide at the boundary in suitable coordinates. In the two dimensional case it is proven in [M2] that (1.1) 
implies that all derivatives of the two metrics coincide at the boundary in suitable coordinates.

The main result of this paper can be easily extended to dimension $n>3$. It is likely that the methods of this paper will give stability results as well as local results near other Riemannian metrics than the euclidian metric. For other type of local results see [C-D-S].

\section{Preliminaries. The main identity}

Assume that we have two metrics $g_{1}$ and $g_{2}$ satisfying

$$
g-e \in C_{(0)}^{k}(\Omega), \quad\|g-e\|_{C^{k}(\bar{\Omega})}<\varepsilon,
$$

with some $k \geq 2$ and $\varepsilon>0$. Assume also that they satisfy (1.1). By (2.1), $g_{1}$ and $g_{2}$ can be extended outside $\Omega$ as $e$ and the so extended metrics belong to $C^{k}\left(\mathbb{R}^{3}\right)$. From now on we will denote by $g_{1}$ and $g_{2}$ the extended metrics.

The Hamiltonian related to $g$, where $g$ is either $g_{1}$ or $g_{2}$, is

$$
H=\frac{1}{2}\left(\sum_{i, j=1}^{3} g^{i j}(x) \xi_{i} \xi_{j}-1\right) .
$$

Given $x^{(0)} \in \Gamma, \xi^{(0)} \in S^{2}$, such that $\nu\left(x^{(0)}\right) \cdot g^{-1} \xi^{(0)}<0$, denote by $x_{g_{j}}, \xi_{g_{j}}$, $j=1,2$ the solution to the Hamiltonian system

$$
\begin{aligned}
\frac{d}{d s} x_{m} & =\sum_{j=1}^{3} g^{m j} \xi_{j}, & \frac{d}{d s} \xi_{m} & =-\frac{1}{2} \sum_{i, j=1}^{3} \frac{\partial g^{i j}}{\partial x_{m}} \xi_{i} \xi_{j}, \quad m=1,2,3, \\
\left.x\right|_{s=0} & =x^{(0)}, & \left.\xi\right|_{s=0} & =\xi^{(0)} .
\end{aligned}
$$

Here $g$ is either $g_{1}$ or $g_{2}$, while the initial conditions are the same for both metrics. We remark that if $\xi^{(0)} \cdot g^{-1} \xi^{(0)}=1$, then $s$ is the arc-length in (2.2). The assumption (1.1) implies the following property.

Lemma 2.1 (see [M1]). Let $g_{1}, g_{2}$ be two Riemannian metrics in $\bar{\Omega}$ with $\bar{\Omega}$ strictly convex with respect to anyone of them and assume $\left.g_{1}\right|_{\Gamma}=\left.g_{2}\right|_{\Gamma}$. Assume also (1.1). Let $x_{g_{m}}, \xi_{g_{m}}, m=1,2$, be the solution of (2.2) with the same initial conditions

$$
x_{g_{1}}(0)=x_{g_{2}}(0)=x^{(0)}, \quad \xi_{g_{1}}(0)=\xi_{g_{2}}(0)=\xi^{(0)} .
$$

Then

$$
x_{g_{1}}(t)=x_{g_{2}}(t) \in \Gamma, \quad \xi_{g_{1}}(t)=\xi_{g_{2}}(t),
$$

where $t$ is the common length of the corresponding geodesics joining $x^{(0)}$ and $x_{g_{1}}(t)=x_{g_{2}}(t)$ provided that $\xi^{(0)} \cdot g^{-1} \xi^{(0)}=1$. 
Proof. Let $x_{g_{1}}$ be the geodesics related to $g_{1}$ defined above. Denote by $s \mapsto y_{g_{2}}(s)$ the geodesics associated to $g_{2}$ joining $x_{g_{1}}(0)$ and $x_{g_{1}}(t) \in \Gamma$, where $t$ is the length of $x_{g_{1}} x_{g_{2}}$. In other words, $y_{g_{2}}(0)=x_{g_{1}}(0), y_{g_{2}}(t)=x_{g_{1}}(t)$. Note, that $t$ is also the length of that geodesic. By [M1, Corollary 2.3], the geodesics $x_{g_{1}}$ and $y_{g_{2}}$ are tangent at the common endpoints. Since $y_{g_{2}}$ solves (2.2) with $g=g_{2}$ and initial data $y_{g_{2}}=x^{(0)}, \xi(0)=\eta^{(0)}$ with some $\eta^{(0)}$, we get that $\eta^{(0)}=\xi^{(0)}$, because the two metrics coincide on the boundary. Therefore, $y_{g_{2}}$ solves (2.2) with $g=g_{2}$ and by the uniqueness of that solution we get that $y_{g_{2}}=x_{g_{2}}$. This proves the lemma.

Consider the Hamiltonian system (2.2) with the following initial conditions

$$
\begin{aligned}
& \frac{d}{d s} x_{m}=\sum_{j=1}^{3} g^{m j} \xi_{j}, \quad \frac{d}{d s} \xi_{m}=-\frac{1}{2} \sum_{i, j=1}^{3} \frac{\partial g^{i j}}{\partial x_{m}} \xi_{i} \xi_{j}, \quad m=1,2,3, \\
& \left.x\right|_{s=-\rho}=(-\rho, z),\left.\quad \xi\right|_{s=-\rho}=(1,0,0) .
\end{aligned}
$$

Here $z \in \mathbb{R}^{2}, \rho>0$ is such that $g=e$ for $|x|>\rho$ and the solution $x=x(s, z)$, $\xi=\xi(s, z)$ depends on the parameter $z$. If $g=e$, then $x=(s, z)=\left(s, z_{1}, z_{2}\right)$. As in [S-U2], from estimate (2.1) we get.

Lemma 2.2. Let $g$ satisfy (2.1). For the solution $x=x(s, z), \xi=\xi(s, z)$ of (2.4) we have

$$
\|x-(s, z)\|_{C^{k-1}}+\|\xi-(1,0,0)\|_{C^{k-1}} \leq C \varepsilon,
$$

with $C>0$ uniform in any compact set.

Introduce new coordinates $y=(s, z)$. Then the map $\Omega \ni x \mapsto y$ is close to $I d$ in the $C^{k-1}$ topology for small $\varepsilon>0$ and therefore is a diffeomorphism. In the new coordinates $g^{-1}=\left(g^{i j}\right)$ will have the form

$$
\left(g^{i j}\right)=\left(\begin{array}{ccc}
1 & 0 & 0 \\
0 & g^{22} & g^{23} \\
0 & g^{23} & g^{33}
\end{array}\right) .
$$

Notice that $g$ would have a similar form, too.

Denote by $\psi_{1}, \psi_{2}$ the maps $x \mapsto y$ related to $g_{1}, g_{2}$, respectively. Instead of $g_{1}, g_{2}$, consider $\tilde{g}_{1}=\psi_{1}^{*} g_{1}$ and $\tilde{g}_{2}=\psi_{2}^{*} g_{2}$, respectively. It is easy to see that $s$ is the length parameter in (2.4) and therefore (1.1) implies $\psi_{1}(\Gamma)=\psi_{2}(\Gamma)$. So, both $\psi_{1}$ and $\psi_{2}$ map $\Omega$ to a new domain $\tilde{\Omega}$. By $(2.3), \psi_{1}=\psi_{2}$ outside $\Omega$. Therefore, (1.1) remains true for $\tilde{g}_{1}, \tilde{g}_{2}$ in $\tilde{\Omega}$ and instead of (2.1) we have

$$
\tilde{g}_{1}-\tilde{g}_{2} \in C_{(0)}^{k-2}(\tilde{\Omega}), \quad\left\|\tilde{g}_{m}-e\right\|_{C^{k-2}(\bar{\Omega})}<C \varepsilon, \quad m=1,2,
$$

with some $C>0$. We aim to prove that $\tilde{g}_{1}=\tilde{g}_{2}$. This would prove the main theorem, because it would imply $\psi^{*} g_{1}=g_{2}$ where $\psi:=\psi_{2}^{-1} \psi_{1}$ would be a diffeomorphism in $\Omega$ fixing the boundary. For the sake of simplicity of notation, let us denote the new metrics again by $g_{1}, g_{2}$ and $\tilde{\Omega}$ by $\Omega$. 
Denote the solution of (2.2) by $x=x\left(s, x^{(0)}, \xi^{(0)}\right), \xi=\xi\left(s, x^{(0)}, \xi^{(0)}\right)$. Let us introduce new notation

$$
X:=(x, \xi)
$$

The solution to (2.2) related to $g_{1}$ and $g_{2}$, respectively, can therefore be written down as $X_{g_{j}}=X_{g_{j}}\left(s, X^{(0)}\right)=X_{g_{j}}\left(s, x^{(0)}, \xi^{(0)}\right)$.

Set $F(s):=X_{g_{2}}\left(t-s, X_{g_{1}}\left(s, X^{(0)}\right)\right)$. Here $t=t\left(X^{(0)}\right)$ is the length of the geodesics issued from $X^{(0)}$ with endpoint on $\Gamma$ and $t$ is independent of $g=g_{1}$ or $g=g_{2}$. Notice that the $x$-component of $F(s)$ may not be in $\Omega$ but belongs to a neighborhood of $\Gamma$ small with $\varepsilon$. By $(2.3), F(0)=X_{g_{2}}\left(t, X^{(0)}\right)=X_{g_{1}}\left(t, X^{(0)}\right)=$ $F(t)$. Thus

$$
\int_{0}^{t} F^{\prime}(s) d s=0
$$

Denote $V_{g_{j}}:=\left(\partial H_{g_{j}} / \partial \xi,-\partial H_{g_{j}} / \partial x\right), j=1,2$. Then

$$
\begin{aligned}
F^{\prime}(s)= & -V_{g_{2}}\left(X_{g_{2}}\left(t-s, X_{g_{1}}\left(s, X^{(0)}\right)\right)\right) \\
& +\frac{\partial X_{g_{2}}}{\partial X^{(0)}}\left(t-s, X_{g_{1}}\left(s, X^{(0)}\right)\right) V_{g_{1}}\left(X_{g_{1}}\left(s, X^{(0)}\right)\right) .
\end{aligned}
$$

We claim that

$$
\begin{aligned}
& V_{g_{2}}\left(X_{g_{2}}\left(t-s, X_{g_{1}}\left(s, X^{(0)}\right)\right)\right)= \\
& \frac{\partial X_{g_{2}}}{\partial X^{(0)}}\left(t-s, X_{g_{1}}\left(s, X^{(0)}\right)\right) V_{g_{2}}\left(X_{g_{1}}\left(s, X^{(0)}\right)\right) .
\end{aligned}
$$

Indeed, (2.9) follows from

$$
\begin{aligned}
0 & =\left.\frac{d}{d s}\right|_{s=0} X\left(T-s, X\left(s, X^{(0)}\right)\right) \\
& =-V\left(X\left(T, X^{(0)}\right)\right)+\frac{\partial X}{\partial X^{(0)}}\left(T, X^{(0)}\right) V\left(X^{(0)}\right), \quad \forall T,
\end{aligned}
$$

after setting $T=t-s$. Therefore, (2.7), (2.8) and (2.9) combined together imply

$$
\int_{0}^{t} \frac{\partial X_{g_{2}}}{\partial X^{(0)}}\left(t-s, X_{g_{1}}\left(s, X^{(0)}\right)\right)\left(V_{g_{1}}-V_{g_{2}}\right)\left(X_{g_{1}}\left(s, X^{(0)}\right)\right) d s=0 .
$$

Relation (2.10) is our basic equality from which we will derive $g_{1}=g_{2}$. If we assume in (2.2) that the initial condition is given at $t=t_{0}$, then (2.10) remains true with the integral taken over $\left(t_{0}, t_{1}\right)$, where $t_{1}-t_{0}$ is the length of the corresponding geodesic.

To make our approach more clear, we will consider a formal linearization of (2.10). In other words, we will formally replace $X_{g_{1}}$ and $X_{g_{2}}$ by $X_{e}$, where $e$ is the euclidian metric, but we will keep $V_{g_{1}}$ and $V_{g_{2}}$. 
Suppose $g=e$. Then $X_{e}=\left(x_{e}, \xi_{e}\right)$ solves $x_{e}^{\prime}=\xi_{e}, \xi_{e}^{\prime}=0$, therefore $V_{e}=$ $(\xi, 0)$. It is easy to see that in this case

$$
X_{e}=\left(\begin{array}{cc}
1 & s \\
0 & 1
\end{array}\right) X^{(0)}, \quad \frac{\partial X_{e}}{\partial X^{(0)}}=\left(\begin{array}{cc}
1 & s \\
0 & 1
\end{array}\right)
$$

Since $V=\left(g^{-1} \xi,-\frac{1}{2} \nabla_{x}\left(g^{-1} \xi\right) \cdot \xi\right)$ (recall that $\left.g^{-1}=\left\{g^{i j}\right\}\right)$, we get the following formal linearization formula for $(2.10)$

$$
\int_{0}^{t}\left(m \xi-\frac{1}{2}(t-s) \nabla_{x}(m \xi) \cdot \xi,-\frac{1}{2} \nabla_{x}(m \xi) \cdot \xi\right)\left(x^{(0)}+s \xi\right) d s=0
$$

where $\left\{m_{i j}\right\}:=\left\{g_{1}^{i j}\right\}-\left\{g_{2}^{i j}\right\}, x^{(0)} \in \Gamma, \xi=\xi^{(0)} \in S^{2}$ and $\xi^{(0)} \cdot \nu\left(x^{(0)}\right)<0$. By (2.5), $m$ has the form

$$
m=\left(\begin{array}{ccc}
0 & 0 & 0 \\
0 & m_{22} & m_{23} \\
0 & m_{23} & m_{33}
\end{array}\right) .
$$

Equating the second components of both sides in (2.12), we get

$$
\int_{0}^{t} \sum_{i, j=2}^{3} \nabla_{x} m_{i j}\left(x^{(0)}+s \xi\right) \xi_{i} \xi_{j} d s=0
$$

for $x^{(0)}$ and $\xi$ as above. This equation easily implies

$$
\sum_{i, j=2}^{3} \eta \hat{m}_{i j}(\eta) \xi_{i} \xi_{j}=0 \quad \text { for } \xi \cdot \eta=0
$$

where $\hat{m}(\eta)$ is the Fourier transform of $m(x)$ extended as 0 outside $\Omega$. Let $p=\left(0, p_{2}, p_{3}\right) \in S^{2}$ be a parameter. Picking

$$
\xi=\xi_{p}(\eta)=\frac{\eta \times p}{|\eta \times p|}=\frac{\left(p_{3} \eta_{2}-p_{2} \eta_{3},-p_{3} \eta_{1}, p_{2} \eta_{1}\right)}{\sqrt{\eta_{1}^{2}+\left(p_{3} \eta_{2}-p_{2} \eta_{3}\right)^{2}}}
$$

we get

$$
\eta \frac{p_{2}^{2} \eta_{1}^{2} \hat{m}_{33}(\eta)+p_{3}^{2} \eta_{1}^{2} \hat{m}_{22}(\eta)-2 p_{2} p_{3} \eta_{1}^{2} \hat{m}_{23}(\eta)}{\eta_{1}^{2}+\left(p_{3} \eta_{2}-p_{2} \eta_{3}\right)^{2}}=0
$$

Choosing $p=(0,1,0)$ yields

$$
\eta \frac{\eta_{1}^{2}}{\eta_{1}^{2}+\eta_{3}^{2}} \hat{m}_{33}(\eta)=0
$$

therefore $m_{33}=0$. Next, setting $p=(0,0,1)$ in $(2.17)$ leads to

$$
\eta \frac{\eta_{1}^{2}}{\eta_{1}^{2}+\eta_{2}^{2}} \hat{m}_{22}(\eta)=0
$$


so $m_{22}=0$. And finally, choosing $p=(0,1,1) / \sqrt{2}$, we obtain

$$
\eta \frac{\eta_{1}^{2} \hat{m}_{33}(\eta)+\eta_{1}^{2} \hat{m}_{22}(\eta)-2 \eta_{1}^{2} \hat{m}_{23}(\eta)}{\eta_{1}^{2}+\left(\eta_{3}-\eta_{2}\right)^{2} / 2}=0
$$

thus $m_{23}=0$.

\section{Proof of the main result}

In this section we prove Theorem 1.1.

Let $\rho>0$ be such that $\bar{\Omega} \subset B_{\rho}:=\{x ;|x|<\rho\}$ and assume that $g_{1}$ and $g_{2}$ are the transformed metrics of the form (2.5) satisfying (2.6), (1.1). Let us extend $g_{1}$ and $g_{2} C^{k-2}$-smoothly in $B_{\rho} \backslash \Omega$ such that for the so extended $g_{j}$ we have $g_{1}=g_{2}$ in $B_{\rho} \backslash \Omega, g_{j}-e \in C_{(0)}^{k-2}\left(B_{\rho}\right), j=1,2$ and $g_{1}, g_{2}$ satisfy (2.6), (1.1) with $\Omega$ replaced by $B_{\rho}$. We can also assume that the first row and the first column of those metrics remain unchanged, so the extended metrics remain of the form (2.5). In other words, we reduce the problem to two new metrics $g_{1}$ and $g_{2}$ in $B_{\rho}$ which satisfy conditions similar to those satisfied by the original $g_{1}, g_{2}$ in $\Omega$ but have special form. Notice that $B_{\rho}$ is strictly convex with respect to $g_{1}, g_{2}$ and for $\varepsilon>0$ small enough there is a unique geodesics (with respect to either metric) joining any two points in $B_{\rho}$. We can further extend those two metrics as $e$ outside $B_{\rho}$. Notice that after those extensions $m=g_{1}^{-1}-g_{2}^{-1}$ extends as zero outside $\Omega$. Instead of solving (2.2), we will solve the same Hamiltonian system for $g=g_{1}$ with modified initial conditions. Assume now that

$$
z \in\left\{x ; x \cdot \xi^{(0)}=0\right\}, \quad \xi^{(0)} \in S^{2},
$$

and solve

$$
\begin{array}{ll}
\frac{d}{d s} x_{m}=\sum_{j=1}^{3} g^{m j} \xi_{j}, & \frac{d}{d s} \xi_{m}=-\frac{1}{2} \sum_{i, j=1}^{3} \frac{\partial g^{i j}}{\partial x_{m}} \xi_{i} \xi_{j}, \quad m=1,2,3 \\
\left.x\right|_{s=-\rho}=x^{(0)}:=z-\rho \xi^{(0)}, & \left.\xi\right|_{s=-\rho}=\xi^{(0)} \in S^{2}, \quad x^{(0)} \cdot \xi^{(0)}=-\rho
\end{array}
$$

where $g$ is either $g_{1}$ or $g_{2}$. We will denote the solution of (3.2) by $x=x_{g}\left(s, z, \xi^{(0)}\right)$, $\xi=\xi_{g}\left(s, z, \xi^{(0)}\right)$. If $g=e$, then $x_{e}=z+s \xi^{(0)}, \xi_{e}=\xi^{(0)}$. For general $g$ satisfying $(2.6)$,

$$
x_{g}=z+s \xi^{(0)}+O(\varepsilon), \quad \xi=\xi^{(0)}+O(\varepsilon) \quad \text { in } C^{k-3} .
$$

Here the $C^{k-3}$ norm is meant with respect to all variables $s, z, \xi^{(0)}$. Here and in what follows $O(\varepsilon)$ will denote various functions with norm bounded by $C \varepsilon$ with a constant $C>0$ uniform in any fixed compact set. Estimate (3.3) follows from Lemma 2.2 for $\xi^{(0)}$ fixed parameter and it is easy to see that it holds also for $\xi^{(0)}$ considered as a variable.

Clearly, for any $\xi^{(0)} \in S^{2},(s, z)$ are Euclidean coordinates in $\mathbb{R}^{3}$. The map

$$
x_{e}=z+s \xi^{(0)} \mapsto x=x_{g}\left(s, z, \xi^{(0)}\right) \in B_{\rho}
$$


is a diffeomorphism for $\varepsilon>0$ small enough, because it is close to $I d$ in the $C^{k-3}$ topology. Denote the Jacobian of this change of variables (related to $g=g_{1}$ ) by $J_{1}\left(x, \xi^{(0)}\right)$.

With this choice of $X_{g_{1}}, X_{g_{2}}$, consider $(2.10)$ with $X^{(0)}=\left(x^{(0)}, \xi^{(0)}\right)=(z-$ $\left.\rho \xi^{(0)}, \xi^{(0)}\right)$. Вy $(3.3)$,

$$
\left\|X_{g_{2}}-\left(\begin{array}{cc}
1 & s \\
0 & 1
\end{array}\right) X^{(0)}\right\|_{C^{k-3}}=O(\varepsilon)
$$

therefore,

$$
\left\|\frac{\partial X_{g_{2}}}{\partial X^{(0)}}-\left(\begin{array}{cc}
1 & s \\
0 & 1
\end{array}\right)\right\|_{C^{k-4}}=O(\varepsilon)
$$

We thus get that in (2.10) we have

$$
\frac{\partial X_{g_{2}}}{\partial X^{(0)}}\left(t-s, X_{g_{1}}\left(s, X^{(0)}\right)\right)=\left(\begin{array}{cc}
1 & t-s \\
0 & 1
\end{array}\right)+O(\varepsilon) \quad \text { in } C^{k-4},
$$

with respect to the variables $s$ and $X^{(0)}$. Here $t$ is such that $t+\rho$ is the length of the geodesics issued from $X^{(0)}=\left(x^{(0)}, \xi^{(0)}\right)$ (with $x^{(0)} \cdot \xi^{(0)}=-\rho$ ) with endpoint $X^{(1)}$ such that $x^{(1)} \in B_{\rho}$ and the geodesics $X^{(0)} X^{(1)}$ crosses $B_{\rho}$. So, (2.10) can be rewritten as

$$
\int_{-\rho}^{t}\left(\left(\begin{array}{cc}
1 & t-s \\
0 & 1
\end{array}\right)+B(s)\right)\left(V_{g_{1}}-V_{g_{2}}\right)\left(X_{g_{1}}\left(s, X^{(0)}\right)\right) d s=0,
$$

where

$$
B(s)=B\left(s, X^{(0)} ; g_{1}, g_{2}\right)=\left(\begin{array}{cc}
B_{11} & B_{12} \\
B_{21} & B_{22}
\end{array}\right)=O(\varepsilon) \quad \text { in } C^{k-4} .
$$

Each block $B_{i j}$ here is a $3 \times 3$ matrix. Recall that $V_{g_{1}}-V_{g_{2}}=\left(m \xi,-\frac{1}{2} \nabla_{x}(m \xi) \cdot \xi\right)$. The left-hand side of (3.7) is a 6-dimensional vector. Let us use the fact that its last 3 components vanish:

$$
\int\left(\frac{\partial m}{\partial x_{j}} \xi \cdot \xi-2\left(B_{21} m \xi\right)_{j}+\sum_{i=1}^{3}\left(B_{22}\right)_{j i} \frac{\partial m}{\partial x_{i}} \xi \cdot \xi\right) d s=0, \quad j=1,2,3
$$

Here we used the fact that $\operatorname{supp} m \subset B_{\rho}$ and therefore we can integrate with respect to $s$ over the whole real line.

Let us Fourier transform (3.8) with respect to $z \in\left\{z \cdot \xi^{(0)}=0\right\}$. Then the dual variable will belong to the same plane

$$
\begin{aligned}
\int_{z \cdot \xi^{(0)}=0} \int e^{-i \eta \cdot z}( & \frac{\partial m}{\partial x_{j}} \xi \cdot \xi-2\left(B_{21} m \xi\right)_{j} \\
& \left.+\sum_{i=1}^{3}\left(B_{22}\right)_{j i} \frac{\partial m}{\partial x_{i}} \xi \cdot \xi\right) d s d S_{z}=0, \quad \eta \cdot \xi^{(0)}=0
\end{aligned}
$$


$j=1,2,3$. Recall that here $\xi^{(0)} \in S^{2}$ is a parameter and $m=m(x), x=$ $x_{g_{1}}\left(s, z, \xi^{(0)}\right), \xi=\xi_{g_{1}}\left(s, z, \xi^{(0)}\right)$. In the integral above we can replace the phase function $\eta \cdot z$ by $\eta \cdot x_{e}=\eta \cdot\left(z+s \xi^{(0)}\right)$. Let us make the change of variables (3.4) in $(3.9)$

$$
\begin{aligned}
\int e^{-i \eta \cdot x_{e}}( & \frac{\partial m}{\partial x_{j}} \xi \cdot \xi-2\left(B_{21} m \xi\right)_{j} \\
& \left.+\sum_{i=1}^{3}\left(B_{22}\right)_{j i} \frac{\partial m}{\partial x_{i}} \xi \cdot \xi\right) J_{1}^{-1} d x=0, \quad \eta \cdot \xi^{(0)}=0,
\end{aligned}
$$

$j=1,2,3$. Here $m=m(x), \xi=\xi\left(x, \xi^{(0)}\right)$ and $x_{e}=x_{e}\left(x, \xi^{(0)}\right)$ is the function inverse to (3.4) with $g=g_{1}$.

Let $p=\left(0, p_{2}, p_{3}\right) \in S^{2}$ be a parameter as in (2.16). Choose $\xi^{(0)}=\xi_{p}(\eta)$ as in (2.16) and let us plug this into (3.10). Clearly, $\xi^{(0)} \cdot \eta=0$ and $\left|\xi^{(0)}\right|=1$. Next, $\xi^{(0)}$ is singular on the line $\eta=s p, s \in \mathbb{R}$. To avoid this singularity, choose a cut-off function $\chi_{p}(\eta) \in C^{\infty}\left(\mathbb{R}^{3} \backslash\{0\}\right)$, that vanishes for $\eta$ near that line, i.e.

$$
\chi_{p}(\eta)= \begin{cases}0, & \text { if } 1-|p \cdot \eta| /|\eta|<\delta / 2 \\ 1, & \text { if } 1-|p \cdot \eta| /|\eta|>\delta\end{cases}
$$

Here $\delta>0$ is a small parameter and we assume also that $\chi_{p}$ is homogeneous of order 0 . After multiplying (3.10) by $\chi_{p}(\eta)$ we obtain

$$
\begin{aligned}
\chi_{p}(\eta) \int e^{-i \varphi_{p}(x, \eta)}( & \frac{\partial m}{\partial x_{j}} \xi \cdot \xi-2\left(B_{21} m \xi\right)_{j} \\
& \left.+\sum_{i=1}^{3}\left(B_{22}\right)_{j i} \frac{\partial m}{\partial x_{i}} \xi \cdot \xi\right) J_{1}^{-1} d x=0, \quad \forall \eta \neq 0,
\end{aligned}
$$

$j=1,2,3$. Here $m=m(x), \xi=\xi\left(x, \xi_{p}(\eta)\right), \varphi_{p}(x, \eta)=\eta \cdot x_{e}\left(x, \xi_{p}(\eta)\right)$ and $\xi, \varphi_{p}$ depend smoothly on $\eta \in \operatorname{supp} \chi_{p} \backslash\{0\}$. Moreover, by (3.3), (3.4), for such $\eta$ and $x$ in a compact set,

$$
\varphi_{p}(x, \eta)=x \cdot \eta+O(\varepsilon) \quad \text { in } S_{k-3}^{1}, \quad \xi=\xi_{p}(\eta)+O(\varepsilon) \quad \text { in } S_{k-3}^{0} .
$$

Here and in what follows we denote by $S_{k}^{m}$ the following class of functions. We say that $a=a(x, y, \xi) \in C^{k}\left(B_{\rho}^{2} \times \mathbb{R}^{3} \backslash\{0\}\right)$ belongs to $S_{k}^{m}$ iff there exists a constant $C \geq 0$, such that

$$
\begin{aligned}
\left|\partial_{x}^{\alpha} \partial_{y}^{\beta} \partial_{\xi}^{\gamma} a(x, y, \xi)\right| & \leq C|\xi|^{m-|\gamma|} \\
\quad \text { for }(x, y) & \in B_{\rho}^{2}, \quad \xi \in \mathbb{R}^{3} \backslash\{0\}, \quad|\alpha|+|\beta|+|\gamma| \leq k .
\end{aligned}
$$

The optimal constant in (3.14) defines a norm in $S_{k}^{m}$. We say that $a=O(\varepsilon)$ in $S_{k}^{m}$ iff $a \in S_{k}^{m}$ and the $S_{k}^{m}$-norm of $a$ is $O(\varepsilon)$, in other words (3.14) holds with $C$ replaced by $C \varepsilon$. We are going to use later [S-U1, Theorem A.1] and [S-U2, 
Proposition 4.1] about boundedness of FIOs and IDOs with amplitudes and phase function of finite smoothness belonging to the class introduced above.

Recall that $\xi\left(x, \xi_{p}(\eta)\right)$ is defined by $\xi\left(x, \xi_{p}(\eta)\right)=\xi_{g_{1}}\left(s, z, \xi_{p}(\eta)\right)$, where $z=$ $z(x), s=s(x)$ are determined by $x=x_{g_{1}}\left(s, z, \xi^{(0)}\right)$ (see (3.4)). Assume that $\xi^{(0)}= \pm e_{1}$. It is easy to see then that $x_{g_{1}}\left(s, z, \pm e_{1}\right)=z \pm s e_{1}, \xi_{g_{1}}\left(s, z, \pm e_{1}\right)=$ $\pm e_{1}$. Since $\eta_{1}=0$ implies $\xi_{p}(\eta)= \pm e_{1}$, for $\eta_{1}=0$ the remainder in (3.13) therefore vanishes and we actually have

$\xi=\xi_{p}(\eta)+\frac{\eta_{1}}{|\eta|} O(\varepsilon)=\frac{\eta_{1}}{\sqrt{\eta_{1}^{2}+\left(p_{3} \eta_{2}-p_{2} \eta_{3}\right)^{2}}}\left[\left(\frac{p_{3} \eta_{2}-p_{2} \eta_{3}}{\eta_{1}},-p_{3}, p_{2}\right)+O_{1}(\varepsilon)\right]$

where $O(\varepsilon)$ and $O_{1}(\varepsilon)$ are in $S_{k-4}^{0}$. Similarly,

$$
B_{21}:=\left(\partial \xi_{g_{2}} / \partial x^{(0)}\right)\left(t-s, X_{g_{1}}\left(s, X^{(0)}\right)\right)
$$

(see (3.7)) vanishes for $\xi^{(0)}= \pm e_{1}$, so after the substitution $\xi^{(0)}=\xi_{p}(\eta)$ we get $B_{21}=\frac{\eta_{1}}{|\eta|} \tilde{B}_{21}$ with $\tilde{B}_{21}=O(\varepsilon)$ in $S_{k-5}^{0}$. Note that $m_{1 j}=m_{j 1}=0, j=1,2,3$, so the first component of $\xi$ plays no role in (3.12). Therefore, the expression in the parentheses in (3.12) can be rewritten as

$$
\begin{aligned}
& \frac{\partial m}{\partial x_{j}} \xi \cdot \xi-2\left(B_{21} m \xi\right)_{j}+\sum_{i=1}^{3}\left(B_{22}\right)_{j i} \frac{\partial m}{\partial x_{i}} \xi \cdot \xi \\
&=\frac{\eta_{1}^{2}}{\eta_{1}^{2}+\left(p_{3} \eta_{2}-p_{2} \eta_{3}\right)^{2}}\left(p_{2}^{2} \frac{\partial m_{33}}{\partial x_{j}}+p_{3}^{2} \frac{\partial m_{22}}{\partial x_{j}}-2 p_{2} p_{3} \frac{\partial m_{23}}{\partial x_{j}}\right. \\
&\left.+\sum_{\alpha, \beta=2}^{3} C_{\alpha \beta}^{(j)} m_{\alpha \beta}+\sum_{i=1}^{3} \sum_{\alpha, \beta=2}^{3} E_{\alpha \beta i}^{(j)} \frac{\partial m_{\alpha \beta}}{\partial x_{i}}\right)
\end{aligned}
$$

where $C_{\alpha \beta}^{(j)}=O(\varepsilon), E_{\alpha \beta i}^{(j)}=O(\varepsilon)$ in $S_{k-5}^{0}$ for $\eta \in \operatorname{supp} \chi_{p}$. Therefore, in (3.12) we can cancel out the factor $\eta_{1}^{2} /\left(\eta_{1}^{2}+\left(p_{3} \eta_{2}-p_{2} \eta_{3}\right)^{2}\right)$ to get

$$
\begin{aligned}
& \chi_{p}(\eta) \int e^{-i \varphi_{p}(x, \eta)}\left(p_{2}^{2} \frac{\partial m_{33}}{\partial x_{j}}+p_{3}^{2} \frac{\partial m_{22}}{\partial x_{j}}-2 p_{2} p_{3} \frac{\partial m_{23}}{\partial x_{j}}\right. \\
& \left.+\sum_{\alpha, \beta=2}^{3} C_{\alpha \beta}^{(j)} m_{\alpha \beta}+\sum_{i=1}^{3} \sum_{\alpha, \beta=2}^{3} E_{\alpha \beta i}^{(j)} \frac{\partial m_{\alpha \beta}}{\partial x_{i}}\right) J_{1}^{-1} d x=0, \quad \forall \eta \neq 0 .
\end{aligned}
$$

Relations (3.13) and the estimates we have on $C_{\alpha \beta}^{(j)}, E_{\alpha \beta i}^{(j)}$ allows us to apply 
Proposition 4.1 in $[\mathrm{S}-\mathrm{U} 2]$ to get

$$
\begin{aligned}
\chi_{p}(\eta) \int e^{-i \varphi_{p}(x, \eta)}( & \sum_{\alpha, \beta=2}^{3} C_{\alpha \beta}^{(j)} m_{\alpha \beta} \\
& \left.+\sum_{i=1}^{3} \sum_{\alpha, \beta=2}^{3} E_{\alpha \beta i}^{(j)} \frac{\partial m_{\alpha \beta}}{\partial x_{i}}\right) J_{1}^{-1} d x=O(\varepsilon\|\nabla m\|) \quad \text { in } L^{2}\left(\mathbb{R}_{\eta}^{3}\right),
\end{aligned}
$$

provided that $k-5=7$. Here $\|\nabla m\|^{2}:=\sum_{i=1}^{3}\left\|\partial m / \partial x_{i}\right\|_{L^{2}}^{2}$ and we have used the Poincaré inequality $\|m\|=\|m\|_{L^{2}\left(B_{\rho}\right)} \leq C\|\nabla m\|$ to estimate the term involving $C_{\alpha \beta}^{(j)} m_{\alpha \beta}$. Hence,

$$
\begin{aligned}
\chi_{p}(\eta) \int e^{-i \varphi_{p}(x, \eta)}\left(p_{2}^{2} \frac{\partial m_{33}}{\partial x_{j}}\right. & \left.+p_{3}^{2} \frac{\partial m_{22}}{\partial x_{j}}-2 p_{2} p_{3} \frac{\partial m_{23}}{\partial x_{j}}\right) J_{1}^{-1} d x \\
& =O(\varepsilon\|\nabla m\|) \text { in } L^{2}\left(\mathbb{R}_{\eta}^{3}\right) .
\end{aligned}
$$

Let us multiply (3.17) by $\exp \left\{i \varphi_{p}(y, \eta)\right\}$ and integrate in $\eta$. Since by [S-U2], Proposition 4.1, this is a continuous operation from $L^{2}$ into $L^{2}\left(B_{\rho}\right)$, we obtain

$$
\begin{aligned}
\iint e^{i\left(\varphi_{p}(y, \eta)-\varphi_{p}(x, \eta)\right)} & \chi_{p}(\eta)\left(p_{2}^{2} \frac{\partial m_{33}}{\partial x_{j}}+p_{3}^{2} \frac{\partial m_{22}}{\partial x_{j}}\right. \\
& \left.-2 p_{2} p_{3} \frac{\partial m_{23}}{\partial x_{j}}\right) J_{1}^{-1} d x d \eta=O(\varepsilon\|\nabla m\|)
\end{aligned}
$$

in $L^{2}\left(\left(B_{\rho}\right)_{y}\right), j=1,2,3$. Next, as in [S-U2], introduce the function $\theta(x, y, \eta)$ by

$$
\theta(x, y, \eta)=\int_{0}^{1}\left(\nabla_{x} \varphi_{p}\right)(x+t(y-x), \eta) d t .
$$

With this choice of $\theta$,

$$
\varphi_{p}(y, \eta)-\varphi_{p}(x, \eta)=(y-x) \cdot \theta(x, y, \eta) .
$$

The function $\theta$ is homogeneous of order 1 in $\eta \in \operatorname{supp} \chi_{p}$ and for such $\eta$ we have $\theta=\eta+O(\varepsilon)$ in $S_{k-4}^{1}$. The equation $\theta=\theta(x, y, \eta)$ can be solved for $\eta$ for $\varepsilon$ small enough and $\eta \in \operatorname{supp} \chi_{p},(x, y) \in B_{\rho}$. The solution $\eta=\eta(x, y, \theta)$ satisfies $\eta=\theta+O(\varepsilon)$ in $S_{k-4}^{1}$ for $\theta$ away from some conic neighborhood of the line $\theta=s p$, $s \in \mathbb{R}$. Next, for the Jacobian $J_{2}:=\operatorname{det}(d \theta / d \eta)$ we have $J_{2}=1+O(\varepsilon)$ in $S_{k-5}^{0}$. After the change $\eta \mapsto \theta$ in (3.18) we get

$$
\begin{gathered}
\iint e^{i(y-x) \cdot \theta} \chi_{p}(\eta(x, y, \theta)) M_{p}(x) J_{1}^{-1} J_{2}^{-1} d x d \theta \\
=O(\varepsilon\|\nabla m\|) \quad \text { in } L^{2}\left(B_{\rho}\right)
\end{gathered}
$$


$j=1,2,3$, where

$$
M_{p}:=p_{2}^{2} \frac{\partial m_{33}}{\partial x_{j}}+p_{3}^{2} \frac{\partial m_{22}}{\partial x_{j}}-2 p_{2} p_{3} \frac{\partial m_{23}}{\partial x_{j}} .
$$

Here $\xi=\xi\left(x, \xi_{p}(\eta(\theta))\right)=\xi_{p}(\theta)+O(\varepsilon)$ in $S_{k-4}^{1}$ for $\theta$ away for a small (with $\varepsilon$ ) conic neighborhood of the line $\theta=s p, s \in \mathbb{R}$. Next, $J_{1}^{-1} J_{2}^{-1}=1+O(\varepsilon)$ in $S_{k-5}^{0}$ for such $\theta$. Let $a(x)$ be a smooth cut-off function supported in $B_{\rho}$ such that $a=1$ on $\operatorname{supp} m$. Then we can rewrite (3.20) as

$$
\begin{aligned}
\iint e^{i(y-x) \cdot \theta} a(y) \chi & (\eta(x, y, \theta)) a(x) M_{p}(x) J_{1}^{-1} J_{2}^{-1} d x d \theta \\
=O(\varepsilon\|\nabla m\|) &
\end{aligned}
$$

$j=1,2,3$. For the amplitude in this oscillating integral we have

$$
a(y) \chi_{p}(\eta(x, y, \theta)) a(x) J_{1}^{-1} J_{2}^{-1}=a(y) \chi_{p}(\theta) a(x)+O(\varepsilon) \quad \text { in } S_{k-5}^{0} .
$$

Moreover, for $\varepsilon>0$ sufficiently small, $\operatorname{supp}_{\theta} \eta(x, y, \theta)$ is away from the singular line $\theta=s p$ for $(x, y) \in B_{\rho}^{2}$. According to Theorem A.1 in [S-U1], if $k-5=$ 7 , this is enough to conclude that we can replace the amplitude in (3.21) by $a(y) \chi_{p}(\theta) a(x)$ and this will result in a remainder term $O(\varepsilon\|\nabla m\|)$, i.e.,

$$
a(y) \iint e^{i(y-x) \cdot \theta} \chi_{p}(\theta) M_{p}(x) d x d \theta=O(\varepsilon\|\nabla m\|) \quad \text { in } L^{2}\left(\mathbb{R}_{y}^{3}\right),
$$

$j=1,2,3$. Multiply $(3.22)$ by $M_{p}(y)$ and integrate in $y$ to get $\left(\hat{M}_{p}, \chi_{p} \hat{M}_{p}\right)=$ $O\left(\varepsilon\|\nabla m\|^{2}\right)$. This yields

$$
\begin{gathered}
\theta\left(p_{2}^{2} \hat{m}_{33}(\theta)+p_{3}^{2} \hat{m}_{22}(\theta)-2 p_{2} p_{3} \hat{m}_{23}(\theta)\right)=O(\sqrt{\varepsilon}\|\nabla m\|) \\
\text { in } L^{2}\left(\left\{1-\frac{|p \cdot \theta|}{|\theta|}>\delta\right\}\right),
\end{gathered}
$$

(compare with (2.17)). Recall that $\delta>0$ was a small parameter.

Choose first $p=\left(0, p_{2}, p_{3}\right)=(0,1,0)$. Then

$$
\theta \hat{m}_{33}(\theta)=O(\sqrt{\varepsilon}\|\nabla m\|) \quad \text { in } L^{2}\left(\left\{\frac{\left|\theta_{2}\right|}{|\theta|}<1-\delta\right\}\right) .
$$

Next, setting $p=(0,0,1)$ yields

$$
\theta \hat{m}_{22}(\theta)=O(\sqrt{\varepsilon}\|\nabla m\|) \quad \text { in } L^{2}\left(\left\{\frac{\left|\theta_{3}\right|}{|\theta|}<1-\delta\right\}\right) .
$$

Let us now set $p=(0,1,2) / \sqrt{5}$ and next $p=(0,2,1) \sqrt{5}$. We get

$$
\begin{aligned}
\theta\left(\hat{m}_{33}(\theta)+4 \hat{m}_{22}(\theta)-4 \hat{m}_{23}(\theta)\right)= & O(\sqrt{\varepsilon}\|\nabla m\|) \\
& \text { in } L^{2}\left(\left\{\frac{\left|\theta_{2}+2 \theta_{3}\right|}{\sqrt{5}|\theta|}<1-\delta\right\}\right),
\end{aligned}
$$




$$
\begin{aligned}
\theta\left(4 \hat{m}_{33}(\theta)+\hat{m}_{22}(\theta)-4 \hat{m}_{23}(\theta)\right)= & O(\sqrt{\varepsilon}\|\nabla m\|) \\
& \text { in } L^{2}\left(\left\{\frac{\left|2 \theta_{2}+\theta_{3}\right|}{\sqrt{5}|\theta|}<1-\delta\right\}\right) .
\end{aligned}
$$

We will prove now that (3.24) holds in the whole $L^{2}$. Indeed, for $\left|\theta_{2}\right| /|\theta|>1-\delta$ we can use (3.25), (3.26) and (3.27) together provided that $\delta>0$ is sufficiently small to get $\theta \hat{m}_{33}=O(\|\nabla m\|)$ for such $\theta$. Therefore,

$$
\theta \hat{m}_{33}(\theta)=O(\sqrt{\varepsilon}\|\nabla m\|) \quad \text { in } L^{2}\left(\mathbb{R}_{\theta}^{3}\right) .
$$

In the same way we get

$$
\theta \hat{m}_{22}(\theta)=O(\sqrt{\varepsilon}\|\nabla m\|) \quad \text { in } L^{2}\left(\mathbb{R}_{\theta}^{3}\right) .
$$

Now, (3.26) and (3.27) combined yield

$$
\theta \hat{m}_{23}(\theta)=O(\sqrt{\varepsilon}\|\nabla m\|) \quad \text { in } L^{2}\left(\mathbb{R}_{\theta}^{3}\right) .
$$

Consequently, by (3.28), (3.29) and (3.30)

$$
\theta \hat{m}(\theta)=O(\sqrt{\varepsilon}\|\nabla m\|) \quad \text { in } L^{2}\left(\mathbb{R}_{\theta}^{3}\right),
$$

thus

$$
\|\nabla m\| \leq C \sqrt{\varepsilon}\|\nabla m\|
$$

with $C$ independent of $\varepsilon, m$ provided that $\varepsilon$ is small enough. Therefore, choosing $\varepsilon<1 / C^{2}$, we get $m=0$ and Theorem 1.1 is proved.

\section{Acknowledgement}

We thank V. Sharafutdinov for making available to us a copy of a preliminary version of the manuscript [C-D-S] after this paper was submitted.

\section{References}

[B] G. Beylkin, Stability and uniqueness of the solution of the inverse kinematic problem in the multidimensional case, J. Soviet Math. 21 (1983), 251-254.

[C] C. Croke, Rigidity and the distance between boundary points, J. Diff. Geom. 33 (1991), 445-464.

[C-D-S] C. Croke, N. S. Dairbekov, and V. A. Sharafutdinov, Local boundary ridigity of a compact Riemannian manifold with curvature bounded above, announcement submitted to "Reports of Russian Academy".

[G-N] M. L. Gerver and N. S. Nadirashvili , An isometricity conditions for Riemannian metrics in a disk, Soviet Math. Dokl. 29 (1984), 199-203.

[Gr] M. Gromov, Filling Riemannian manifolds, J. Diff. Geometry 33 (1991), 445-464.

[G] V. Guillemin, Sojourn times and asymptotic properties of the scattering matrix, Proceedings of the Oji Seminar on Algebraic Analysis and the RIMS Symposium on Algebraic Analysis (Kyoto Univ., Kyoto, 1976). Publ. Res. Inst. Math. Sci. 12 (1976/77), 69-88.

[M1] R. Michel, Sur la rigidité imposée par la longueur des géodésiques, Invent. Math. 65 (1981), 71-83.

[M2] — Restriction de la distance géodésique à un arc et rigidité, Bull. Soc. Math. France 122 (1994), 435-442. 
[Mu] R. G. Mukhometov, The reconstruction problem of a two-dimensional Riemannian metric, and integral geometry, (Russian), Dokl. Akad. Nauk SSSR 232 (1977), 32-35.

[Mu-R] R. G. Mukhometov and V. G. Romanov, On the problem of finding an isotropic Riemannian metric in an n-dimensional space, (Russian), Dokl. Akad. Nauk SSSR 243 (1978), 41-44.

[O] J. P. Otal, Sur les longuer des géodésiques d'une métrique a courbure négative dans le disque, Comment. Math. Helv. 65 (1990), 334-347.

[Sh] V. A. Sharafutdinov, Integral geometry of tensor fields, VSP, Utrech, the Netherlands (1994).

[S-U1] P. Stefanov and G. Uhlmann, Inverse backscattering for the acoustic equation, SIAM J. Math. Anal. 28 (1997), 1191-1204.

[S-U2] _ Stability estimates for the hyperbolic Dirichlet to Neumann map in anisotropic media, J. Funct. Anal. (to appear).

[Sy-U] J. Sylvester and G. Uhlmann, Inverse problems in anisotropic media, Contemp. Math. 122 (1991), 105-197.

Institute of Mathematics, Bulgarian Academy of Sciences, 1113 Sofia, BUlgariA

E-mail address: stefanov@math.washington.edu

Department of Mathematics, University of Washington, Seattle, WA 98195, USA

E-mail address: gunther@math.washington.edu 